\title{
DÜBLIN
}

Technological University Dublin

ARROW@TU Dublin

Articles

School of Food Science and Environmental

Health

2008-09-30

\section{Hand-Held Flyback Driven Coaxial Dielectric Barrier Discharge: Development and Characterization}

\author{
Victor J. Law \\ Dublin City University \\ Vladimir Milosavljevic \\ Technological University Dublin, vladimir.milosavljevic@tudublin.ie \\ Neil O'Connor \\ Dublin City University
}

See next page for additional authors

Follow this and additional works at: https://arrow.tudublin.ie/schfsehart

Part of the Dynamics and Dynamical Systems Commons, Electrical and Electronics Commons, Electronic Devices and Semiconductor Manufacturing Commons, Plasma and Beam Physics Commons, Signal Processing Commons, and the VLSI and Circuits, Embedded and Hardware Systems Commons

\section{Recommended Citation}

V. Law, et al., (2008) Hand-held Flyback driven coaxial dielectric barrier discharge: development and characterization. Review of scientific instruments 79/9, 094707 (2008) DOI http://dx.doi.org/10.1063/ 1.2988833

This Article is brought to you for free and open access by the School of Food Science and Environmental Health at ARROW@TU Dublin. It has been accepted for inclusion in Articles by an authorized administrator of ARROW@TU Dublin. For more information, please contact arrow.admin@tudublin.ie, aisling.coyne@tudublin.ie, gerard.connolly@tudublin.ie.

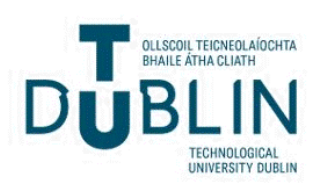




\section{Authors}

Victor J. Law, Vladimir Milosavljevic, Neil O'Connor, James F. Lalor, and Steven Daniels

This article is available at ARROW@TU Dublin: https://arrow.tudublin.ie/schfsehart/192 


\title{
Handheld Flyback driven coaxial dielectric barrier discharge: Development and characterization
}

\author{
V. J. Law, ${ }^{1}$ V. Milosavljević, ${ }^{2, a)}$ N. O'Connor, ${ }^{3}$ J. F. Lalor, ${ }^{4}$ and S. Daniels ${ }^{5}$ \\ ${ }^{1}$ National Center of Plasma Science and Technology (NCPST), Dublin City University, Dublin 9, Ireland \\ ${ }^{2}$ School of Physics and NCPST, Dublin City University, Dublin 9, Ireland and Faculty of Physics, University \\ of Belgrade, P.O. Box 368, Belgrade, Serbia \\ ${ }^{3}$ School of Electronic Engineering and NCPST, Dublin City University, Dublin 9, Ireland \\ ${ }^{4}$ NCPST, Dublin City University, Dublin 9, Ireland \\ ${ }^{5}$ School of Electronic Engineering and NCPST, Dublin City University, Dublin 9, Ireland
}

(Received 14 February 2008; accepted 22 August 2008; published online 30 September 2008)

\begin{abstract}
The development of a handheld single and triple chamber atmospheric pressure coaxial dielectric barrier discharge driven by Flyback circuitry for helium and argon discharges is described. The Flyback uses external metal-oxide-semiconductor field-effect transistor power switching technology and the transformer operates in the continuous current mode to convert a continuous dc power of $10-33 \mathrm{~W}$ to generate a $1.2-1.6 \mathrm{kV} 3.5 \mu$ s pulse. An argon discharge breakdown voltage of $\sim 768 \mathrm{~V}$ is measured. With a $50 \mathrm{kHz}$, pulse repetition rate and an argon flow rate of $0.5-10$ argon slm (slm denotes standard liters per minute), the electrical power density deposited in the volume discharge increases linearly at a rate of $75 \pm 20 \% \mathrm{~mW} / \mathrm{cm}^{3}$ per $1 \mathrm{slm}$ of gas. Electrical power transfer efficiency between the secondary Flyback coil and the discharge volume increases from $0.1 \%$ to $0.65 \%$. Neutral argon gas forced convection analysis yields a similar energy loss rate to the electrical discharge process. Optical emission spectroscopy studies of the expanding discharge plume into ambient air reveal that the air climatically controls the plume chemistry to produce an abundance of neutral argon atoms and molecular nitrogen. (C) 2008 American Institute of Physics. [DOI: $10.1063 / 1.2988833$ ]
\end{abstract}

\section{INTRODUCTION}

The atmospheric pressure coaxial dielectric barrier discharge (DBD) has been established for nonthermal plasma processing for more than 20 years. ${ }^{1-3}$ Their potential for direct impact on society in the guise of surface modification of engineering materials and the destruction of microbial pathogens on contaminated surfaces are now becoming apparent. Recently atmospheric pressure glow discharges (APGD) in the form of plasma pencil, ${ }^{4}$ needle, ${ }^{5,6}$ jet, $^{7}$ and torch $^{8}$ have been reported to have low electrical power consumption. The common feature of the APGD is that they have a volume discharge that acts as a source of ion species and an expanding plume that is driven by the flow of gas passing through the chamber volume. The drive circuitry typically employs a variety of sinusoidal or pulse power technology; some of which have fixed frequencies (13.56 MHz) (Refs. 4-6) or variable frequencies $(7 \mathrm{kHz}$ and $1 \mathrm{MHz})$ (Refs. 7-9). Within this body of work ${ }^{7-10}$ and within the water and food purification sectors, ${ }^{11,12}$ it is commonly believed that continuous power switching technology has better power conversion efficiencies than sinusoidal excitation.

Our Flyback power switching technology has been investigated as an alternative low cost power source and simple self-resisting technology for the coaxial DBD. ${ }^{13,14}$ With no matching network and the outer electrode at ground

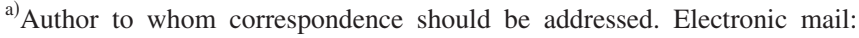
vladimir@physics.dcu.ie.
}

potential, the Flyback design enables a low volume discharge, with an expanding plume to be manufactured as a handheld device. This enables an energetic plasma plume chemistry to be focused on a microbial pathogen contaminated surface.

This paper presents a study of the Flyback design in the form of an externally driven Flyback circuitry. The main working gas studied here is $99.9 \%$ pure argon and the plasma plume expands into ambient air $(78 \%$ molecular nitrogen, $21 \%$ molecular oxygen, with $1 \%$ of other constituent gases). A helium volume discharge is also compared to study the high pressure Penning ionization process (impact of high energy helium metastables on nitrogen impurities in the helium working gas). ${ }^{15}$ The technology developments described here include continuous power switching of a $1-1.6 \mathrm{kV} 3.5 \mu \mathrm{s}$ pulse at an $8-80 \mathrm{kHz}$ pulse repetition rate (PRR) to deliver an applied power of $30-40 \mathrm{~W}$ at the coaxial dielectric barrier anode.

In this paper we characterize the afterglow of an atmospheric pressure of $99.9 \%$ pure helium and $99.9 \%$ pure argon plasma using optical emission spectroscopy. The approach is based on monitoring emission of nitrogen neutrals and ionized molecular bands with respect of the plasma operating conditions, such as flow rate, power, etc. The nitrogen is present as an impurity from the atmosphere in our plasma discharge. Emissions of other impurities are not so significant, i.e., molecular oxygen has a very weak emission spectrum from 200 to $850 \mathrm{~nm}$ and atomic oxygen has been observed only by the spectral line (triplet) at $777 \mathrm{~nm}$. On the 


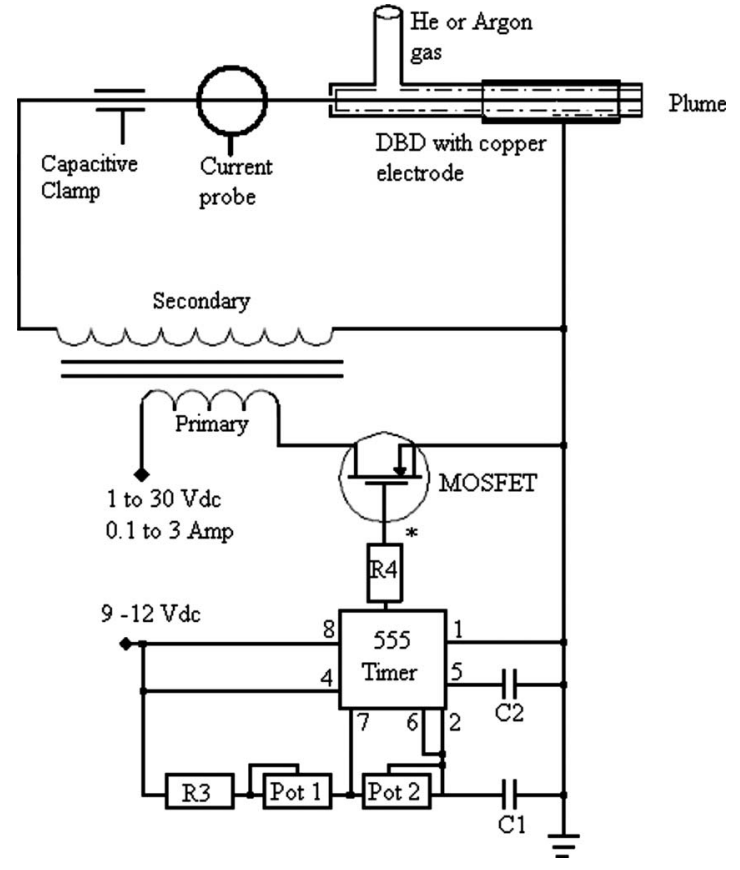

FIG. 1. Schematic of external Flyback drive circuit for helium and argon discharges.

other hand the emission of molecular nitrogen at $337 \mathrm{~nm}$ is very strong and depends on plasma operating conditions. Moreover, in this spectral region there are no emissions from helium and argon atoms. (Therefore our spectrometer has resolved the spectrum very well).

\section{EXPERIMENTAL SETUP}

\section{A. External Flyback drive circuit for argon}

This section describes the external drive circuitry for the generation of an argon discharge using 0.5-10 slm (slm denotes standard liters per minute) of argon as the working gas. In this circuitry the switching frequency element is decoupled from the Flyback power supply (see Fig. 1). Here the switching frequency is controlled by a LM555 timer that is wired in the astable operation mode with a $9 \mathrm{~V} \mathrm{dc}$ supply at $0.2 \mathrm{~A}$. In this mode, the free running frequency and timer duty cycle are controlled by two external potentiometers (Pot1 and Pot2, each adjustable to a value of $0-10 \mathrm{k} \Omega$ ) and one capacitor $\left(C_{1}=10 \mathrm{nF}\right)$. With the addition of a limiting resistor $\left(R_{3}\right)$ the external capacitor charges through potentiomters 1 and 2 and discharges through potentiometer 2. With this knowledge the timer frequency is defined by Eq. (1). In this equation the value of 1.44 is the LM555 circuit timing constant and for $10 \mathrm{k} \Omega$ potentiometers the frequency ranges between 8 and $100 \mathrm{kHz}$.

$$
f \approx \frac{1}{T}=\frac{1.44}{\left(\operatorname{Pot}_{1}+\operatorname{Pot}_{2}\right) C_{1}} .
$$

The variable duty cycle $(D)$ of the circuit is thus defined by the ratio of the two potentiometers as in Eq. (2). The ratio of the time duration when the output pulse is high to the total period is known as the duty cycle. The duty cycle expressed as a percentage can be calculated with the formula:

$$
D \approx \frac{\operatorname{Pot}_{1}+\text { Pot }_{2}}{\text { Pot }_{1}+2 \text { Pot }_{2}} .
$$

Two International Rectifiers metal-oxide-semiconductor field-effect transistors (MOSFETs) have been tested in the external drive circuit. ${ }^{9,16}$ These are the IRFZ340EPBF and the IRF840. The main difference between them is that the IRF840 has a higher drain to source voltage $(500 \mathrm{~V}$, as compared to $50 \mathrm{~V}$ ) and it can be driven by a LM555 timer. A feature of note for the IRFZ340EPBF (and the IRF540A, see Ref. 8) is that to ensure efficient switching of the MOSFET gate capacitance, especially when considering working at high switching frequencies, the circuit is improved by adding a MOSFET driver (2N2222 silicon switching transistor) at the point ${ }^{*}$ in Fig. 1.

To maintain switching efficiency, minimize heating of the MOSFET junction and ensure oscillation suppression of the square wave trigger signal, the timer gate drive resistor $\left(R_{4}\right)$ is set to $4.7 \Omega$. This is because the resistance causes the gate voltage to rise slowly due to the $R$ - $C$ filter). Hence the value of $R_{4}$ determines the speed at which the magnetic field collapses in the transformer core: the greater the speed of collapse, the greater the induced voltage. With the MOSFET mounted on an extruded aluminum heat sink $(H=50 \mathrm{~mm})$, $W=50 \mathrm{~mm}$, and $D=50 \mathrm{~mm}$, the circuit topology has been utilized in the laboratory for many months for the four DBD chambers described here.

Finally the voltage and current waveforms are captured at the secondary coil output using a capacitive clamp that has a voltage division ratio of 30:1 (Refs. 13 and 14) and a Pearson current monitor (model 2877) that has volt-toampere ratio of $1: 1$.

The external driven circuit operation is as follows. With the primary coil of the Flyback transformer driven by a $(0-30 \mathrm{~V}$ and $0-3 \mathrm{~A}$; maximum power $=60 \mathrm{~W})$ Thurlby Thandar Instruments dc supply, the MOSFET is abruptly switched on and off by the timer output voltage level. During the on period the MOSFET conducts through the primary coil causing an induced magnetic current in the secondary coil, which is in phase with the source voltage. When the MOSFET is turned off the magnetic field in the core, and secondary coil, collapses causing the generation of a large voltage pulse in the secondary coil followed by an induced current that lags behind by $90^{\circ}$. When the MOSFET is not fully turned off this voltage-current behavior forms a ringdown waveform.

A typical example of the secondary coil voltage and current switching waveform, for an argon discharge is shown in Fig. 2. Although not to amplitude scale in this figure, the $9 \mathrm{~V}$ timer trigger pulse is also shown. Here it can be seen that a primary voltage pulse is triggered by the timer square wave falling edge. The primary voltage pulse has a peak voltage of $1.4 \mathrm{kV}$ with a rise time of $\sim 1.5 \mu \mathrm{s}$ and pulse width of $3.5 \mu$ s. It can also be seen that the magnetic current pulse lags behind the voltage pulse by some $90^{\circ}$ and reaches a peak of $0.18 \mathrm{~A}$. Within both the primary voltage and magnetic current pulse there are two regions of distortion: one in the voltage rising (current falling) period and one in the voltage falling (current rising) period. These voltage and current 


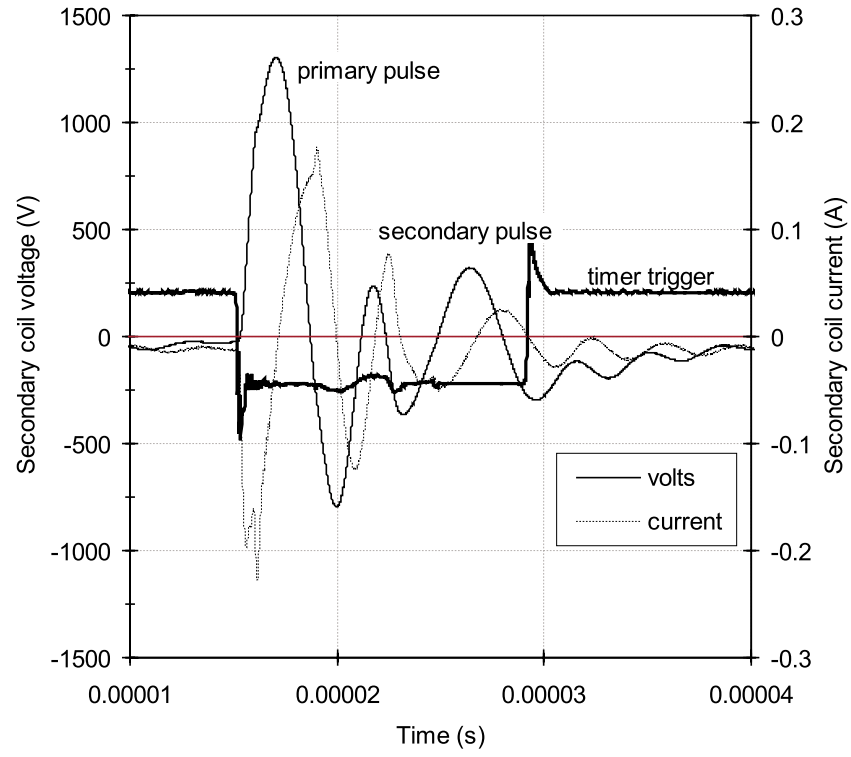

FIG. 2. (Color online) Secondary coil voltage and current waveform $\left(90^{\circ}\right.$ lag) for a timer square-wave frequency of $20 \mathrm{kHz}$. The data were taken on the ceramic DBD version with the following conditions: $\operatorname{argon}=5 \mathrm{slm}$; dc power $=28.5 \mathrm{~W}(19 \mathrm{~V} \times 1.5 \mathrm{~A})$.

distortions are aligned in time, each being close to the peak of the pulse, with a time delay of $\sim 1.5 \mu$ s from the zero crossing point. The remaining voltage and current waveforms dissipate, initially with a nonequal period oscillations and then as an equal period oscillations before returning to zero. This variable ringing is due to a change in Flyback transformer operational mode (continuous and discontinuous) and its interaction with the MOSFET. The continuous mode is when total ampere turns do not dwell at zero, and the discontinuous mode is when the instantaneous ampere turns dwell at zero. The regions of distortion due to the time varying plasma load, and energy transfer between continuous-to-discontinuous modes of the Flyback transformers are described in further detail in Secs. III C and III D. The timer now turns on and the on-off cycle repeats itself until the timer is finally switched off. A further feature of note is when the timing frequency is below $20 \mathrm{kHz}$, vibrations from the circuitry can often be heard as a highpitched whine.

\section{B. Flyback transformer}

The role of the Flyback transformer is to take electrical energy from the drive circuit and store it in a magnetic field, subsequently transferring the magnetic energy to the dielectric barrier and the drive circuit as electrical energy. This release of stored magnetic energy, or freewheel energy, generates the plasma discharge within the dielectric barrier volume when the transistor is in its off period.

The Flyback transformer behavior is more like a multiwinding coupled inductor, unlike a true transformer, wherein magnetic energy storage is undesirable. The inductor current (continuous or discontinuous mode) has a major impact on the circuit performance. The transformer itself is made from a soft ferrite gapped C-core, with a discrete air gap of 0.5 $\mathrm{mm}$. The discrete air gap forms a nonmagnetic discontinuity in the magnetic circuit. The air gap therefore adds reluctance (electric resistance) to the magnetic circuit, and its capacity to store energy. These transformer cores can be obtained from a cathode ray tube of a computer monitor. The transformer windings are made from $0.75 \mathrm{~mm}$ diameter enameled copper wire: five turns on the primary coil, and three turns for the pickup coil in the case of the self-resonating circuit. For the secondary, 105 turns (30 turns per layer $\times 3.5$ layers) are wound on to a polytetrafluoroethylene (PTFE) bobbin. To prevent voltage breakdown the bobbin has a $1 \mathrm{~mm}$ thick wall between the coil and ferrite and secondary windings are encapsulated in insulating transformer varnish. With the complete bobbin positioned over the magnetic air gap the entire magnetic force introduced by the coil is dropped across the gap and within the secondary windings. In this configuration the core provides an easy return path for the magnetic flux with minimal magnetic fields outside the core or windings.

As the secondary coil has one end grounded, its inductance $\left(L_{\text {coil }}\right)$ and self-capacitance (shunt capacitance) behaves as a resonant tank circuit with a resonance above $200 \mathrm{kHz}$. Transmitted power from the primary coil to the secondary will therefore be at maximum when the drive frequency equals the resonance conditions on the secondary coil. The secondary coil also has a shunt capacitance from the dielectric barrier chamber and transmission line $\left(C_{\mathrm{db}}\right)$, and the plasma $\left(C_{p}\right)$ when present. The effective resonant frequency of the secondary coil $\left(f_{\text {rez }}\right)$ is then represented by Eq. (3).

$$
f_{\text {rez }}=\frac{1}{\sqrt{2 \pi L_{\text {coil }}\left(C_{\mathrm{db}}+C_{p}\right)}} .
$$

An inspection of Eq. (3) reveals that a frequency measurement of the secondary coil will always be higher with respect to the additional capacitance of the dielectric barrier and the time varying plasma discharge impedance. The complete tank circuit is therefore frequency pulled when under plasma load conditions. A frequency pulling value of $700 \mathrm{~Hz}$ has been observed in the self-resonant circuit and as reported in Ref. 14. Furthermore the secondary voltage is not related to the primary-to-secondary turn's ratio. In this respect the Flyback design only requires some 120 turns on the secondary coil to produce helium or argon discharges, as compared to 400 turns on secondary in the case of standard transformer design for the production of helium discharge.

\section{DBD chamber design}

This section describes the development of the Flyback DBD chamber. The prototype is presented in Refs. 13 and 14. These design iterations are based on a discharge volume $\left(\pi r^{2} h\right)$ of $\sim 0.25$ cubic centimeters and encompass both material and electrical design.

\section{Hiden Analytical version}

The Hiden Analytical version is made from the original Hiden Analytical atmospheric pressure plasma jet that is driven at $13.56 \mathrm{MHz}$. The electrical and gas housing is constructed from a $\frac{1}{4}$ in. polyethylene Swagelok tee. The two in-line ports form the electrical input and the DBD, one port on the tee forms a gas inlet port. The DBD is formed from a 


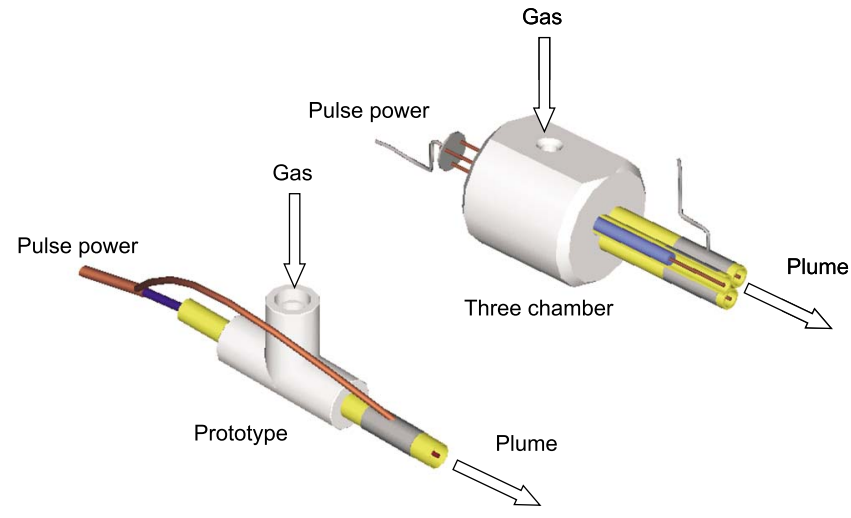

FIG. 3. (Color online) Schematic rendering of single and triple chamber versions.

$6.5 \mathrm{~mm} \mathrm{OD} \times 5 \mathrm{~mm} \mathrm{ID} \times 130 \mathrm{~mm}$ length quartz tube. The outer grounded electrode is wrapped in copper foil. The inner coaxial electrode is constructed from a re-entrant ceramic tube $(3 \mathrm{~mm}$ OD $\times 1.5 \mathrm{~mm}$ ID) that aligns a $1 \mathrm{~mm}$ diameter tungsten wire along the length of the DBD glass tube. An additional $\frac{1}{8}$ in. polyethylene Swagelok tee adds a second gas port, and takes the tungsten wire out of the device for connection to the open circuit end of the secondary winding via $1.2 \mathrm{~m}$ of RG58 transmission line.

This works up to a continuous applied de power of $20 \mathrm{~W}$ $(10 \mathrm{~V} \times 2 \mathrm{~A})$, above this level the quartz nozzle starts to fragment leading after arcing to the ground electrode. Using this reactor, molecular beam mass spectroscopy studies have shown that for a helium discharge the helium ions are quenched in the expanding plume by the ambient gas. For moist air, this quenching results in the formation of positive ion $\left[\mathrm{H}_{3} \mathrm{O}^{+}\left(\mathrm{H}_{2} \mathrm{O}\right) n\right]$ and negative ion $\left[\mathrm{OH}^{-}\left(\mathrm{H}_{2}\right)\right]$ hydrated cluster chemistries where $n$ can be up to 18 depending on the helium gas flow rate. ${ }^{17}$ A number of these chambers are now employed as a atmospheric pressure plasma test sources for atmospheric molecular beam mass spectrometry at Hiden Analytical Ltd. ${ }^{18}$

\section{E. Ceramic version}

The alumina $\left(\mathrm{Al}_{2} \mathrm{O}_{3}\right)$ ceramic version differs from the Hiden version in that it has only one gas port and is made from $99.8 \%$ alumina ceramic (see Fig. 3). The construction is as follows. The electrical and gas housing is constructed from a polyethylene $\frac{1}{4}$ in. Swagelok tee. The two in-line ports form the electrical input and the DBD, the tee leg becomes the helium gas inlet. The DBD is formed from a $5 \mathrm{~mm}$ $\mathrm{OD} \times 4 \mathrm{~mm}$ ID $\times 90 \mathrm{~mm}$ length ceramic tube. The outer grounded electrode is wrapped in copper foil. The inner coaxial electrode is constructed from a re-entrant ceramic tube ( $3 \mathrm{~mm} \mathrm{OD} \times 1.5 \mathrm{~mm} \mathrm{ID)}$ that holds the $1 \mathrm{~mm}$ diameter Tungsten wire. The tungsten wire extends the length of the main ceramic tube and extends into DBD space up to the nozzle. Here again the tungsten wire is connected to the open circuit end of the secondary winding via $1.2 \mathrm{~m}$ of RG58 transmission line.

This design replaces the quartz dielectric material for a machinable ceramic. This innovation allows greater flexibility in physical design geometries, improved thermal stability
(1950 ${ }^{\circ} \mathrm{C}$ as compared to $573{ }^{\circ} \mathrm{C}$ for quartz), and improved charge storage within the applied electromagnetic field due to the higher relative dielectric constant of the alumina ceramic (9.6) as compared to quartz (4.2). With this design, tests have shown that the argon and helium plumes can reach $15 \mathrm{~mm}$ in length for gas flow rates of $1-10 \mathrm{slm}$. The chamber can handle thermal temperatures of $80{ }^{\circ} \mathrm{C}$ and electrical power up to a continuous applied dc power of $40 \mathrm{~W}$ $(20 \mathrm{~V} \times 2 \mathrm{~A})$.

\section{F. Three-chamber ceramic version}

The three-chamber version is a development of the alumina ceramic version (see Fig. 3). Here three ceramic tubes $(5 \mathrm{~mm} \mathrm{OD} \times 4 \mathrm{~mm} \mathrm{ID} \times 90 \mathrm{~mm}$ length) are placed together side by side. A PTFE body is employed to hold the ceramic assembly together and provide electrical and two gas connections. The internal structure of the body is machined to ensure equal working gas flow to all three dielectric chambers. The three re-entrant ceramic tubes $(3 \mathrm{~mm}$ OD $\times 1.5 \mathrm{~mm} \mathrm{ID}$ ) that hold the $1 \mathrm{~mm}$ diameter copper wires enter from the rear of the housing. The three copper wires run the length of the device and are soldered together and then connected to the open circuit end of the Flyback secondary coil via $1.2 \mathrm{~m}$ of RG58 transmission line.

The additional two chambers require that three times as much gas flow is used, as compared to the single chamber versions. Equal gas flow to each chamber requires a second gas input on the opposite side of the housing body to maintain similar plume conditions to that of the single chamber versions. With the addition of two chambers the expanding plume now has an effective diameter of $1.5 \mathrm{~cm}$ when projected on to a glass side at a distance of $1 \mathrm{~cm}$.

\section{RESULTS AND DISCUSSION}

All versions for the Flyback coaxial DBD have been tested with helium and argon. The outcomes of these experiments are tabulated in Table I. The results reveal that all four chamber versions do produce a helium volume discharge and plume. Both the single and three chamber ceramic versions are the only chambers that have produced an argon volume discharge and plume when the IRF840 MOSFET is used. The ceramic version also has better thermal and power handling properties when compared to the prototype and the Hiden version.

\section{A. Neutral gas residence time effect}

Visual characterization of the ceramic DBD volume and plume has been performed as a function of neutral volumetric gas flow rate and residence time $(\tau)$ within the discharge volume. The value of $\tau$ is calculated in the standard way: ${ }^{19}$ $\tau=P V / Q$, where $P=1$ atmosphere, $V$ is the discharge volume in liters and, $Q$ is the flow rate express in $\operatorname{sim}$. To obtain this characterization, the nozzle of the dielectric barrier is placed $1.5 \mathrm{~cm}$ distance from a grounded surface and the plume interaction observed. Figure 4 shows the calculated value of $\tau$ (expressed in seconds) as the argon gas flow is varied from 0 to $10 \mathrm{slm}$ through the ceramic dielectric barrier volume $\left(0.00025 l\right.$, or $\left.0.25 \mathrm{~cm}^{3}\right)$. Superimposed on this curve are 
TABLE I. Electrical comparison between DBD chambers and drive circuits.

\begin{tabular}{|c|c|c|c|c|}
\hline $\begin{array}{l}\text { Chamber } \\
\text { design }\end{array}$ & Drive circuit & $\begin{array}{l}\text { Helium } \\
\text { plasma }\end{array}$ & $\begin{array}{l}\text { Argon } \\
\text { plasma }\end{array}$ & Notes \\
\hline Hiden & External (IRFZ340) & Yes & No & No argon plasma operation \\
\hline Single chamber ceramic & External (IRFZ340) & Yes & No & Voltage damage of MOSFET \\
\hline Three chamber ceramic & External (IRFZ340) & Yes & No & Quartz nozzle fragments at $2 \mathrm{~A}^{\mathrm{b}}$ \\
\hline Single chamber ceramic & External (IRF840) & Yes & Yes & Stable argon plasma operation \\
\hline Three chamber ceramic & External (IRF840) & Yes & Yes & Stable argon plasma operation \\
\hline
\end{tabular}

${ }^{\mathrm{a} A f t e r} 1 \mathrm{~min}$ of argon plasma operation.

${ }^{\mathrm{b}} \mathrm{No}$ argon plasma operation.

three characteristic visual observations of the plume morphology. The correlation reveals that at a flow rate of $0.5 \mathrm{slm}$ $(\tau=0.025 \mathrm{~s})$ the discharge appears to remain within the chamber; in the 1-3 the slm region $(\tau=0.01-0.005 \mathrm{~s})$ the plume forms a stable arc or single filament of some 1-2 mm in diameter and; above the $3 \operatorname{slm}(\tau>0.005 \mathrm{~s})$ the plume forms unstable multiple filaments.

The range of neutral gas residence times within the chamber are some 10-100 times greater than the pulse drive period (1/pulse repetition rate). From this it may be deduced that preconditioning ${ }^{9}$ at the ceramic surface from an early pulse does not have a significant role in the plume production. Even though we cannot measure the pressure drop or viscosity, the calculation of the ratio of the inertial forces to the viscous forces within the dielectric barrier chamber tells us the argon flow is changing from laminar flow at low flows $(0.5-5 \mathrm{slm})$ to turbulent flow at high flows $(5-10 \mathrm{slm})$ with a characteristic Reynolds numbers of $\mathrm{Re}=407-2000$ to 20004100 , respectively. Mathematically $\mathrm{Re}$ is defined in

$$
\operatorname{Re}=\frac{v_{s} \lambda}{\mu / \rho} .
$$

In Eq. (4), $\rho$ is the argon density, $v_{s}$ is the argon velocity within the dielectric barrier chamber, $\lambda$ is the characteristic chamber dimension (diameter), and $\mu$ is the argon viscosity. With an estimated maximum pressure drop of a few millibars

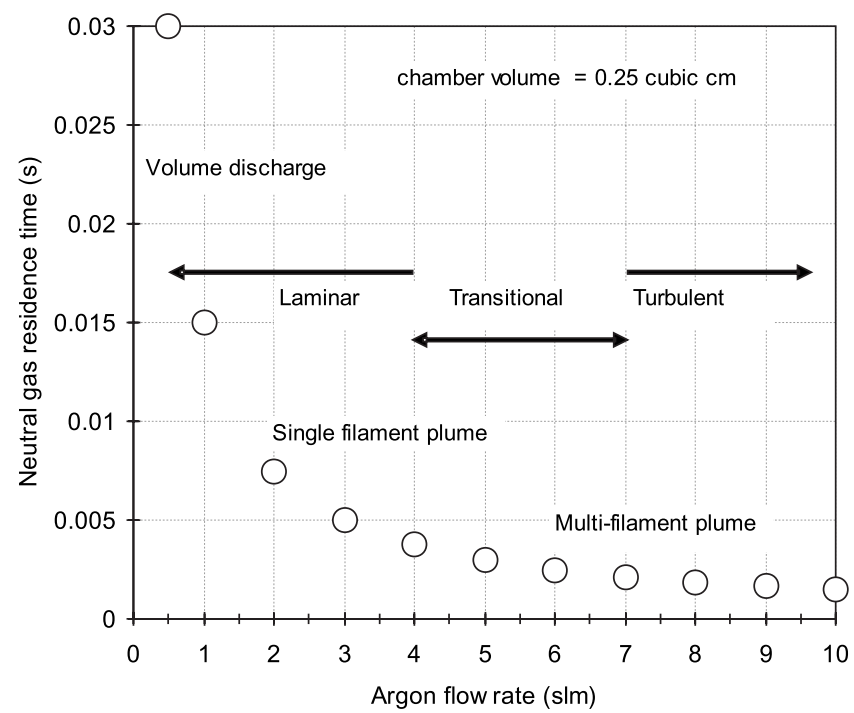

FIG. 4. Comparison between argon gas flow rate and appearance of expanding argon plume upon a copper surface. for these conditions it is reasonable to assume that the change in the flow regime within the chamber and at the nozzle is affecting the electrochemical mechanisms within the plume. This observation needs more investigation over a wider set of parameters. Using the Hiden chamber, however, it has been shown that for similar helium flow rates, heterogeneous mixing with ambient moist air has an impact on the plume chemistry. ${ }^{17}$

\section{B. Secondary coil power performance}

As the primary pulse width is fixed by the speed of collapses of the magnetic field in the secondary coil it is important to have a knowledge of the peak voltage, peak current, and an estimate of the average power as a function of pulse PRR.

Figure 5 shows the measured peak voltage and peak current for the primary $3.5 \mu \mathrm{s}$ pulse as a function of pulse repetition rate for a continuous applied dc power of $30 \mathrm{~W}$ $(20 \mathrm{~V} \times 1.5 \mathrm{~A})$. The power in the primary pulse can be derived by integrating the area under the voltage curve and the two portions of the current pulse curve within the primary voltage pulse width. The $V_{\mathrm{rms}}$ and $I_{\text {rms }}$ integrations are performed by using a trapezoidal pulse shape where the top $(T)$ and bottom $(B)$ are fitted to the voltage and current waveforms. This process is mathematically represented in Eq. (5), where $X_{\mathrm{rms}}$ and $X_{p}$ are used for voltage and current values. The product of the respective $V_{\mathrm{rms}}$ and $I_{\mathrm{rms}}$ values yield a

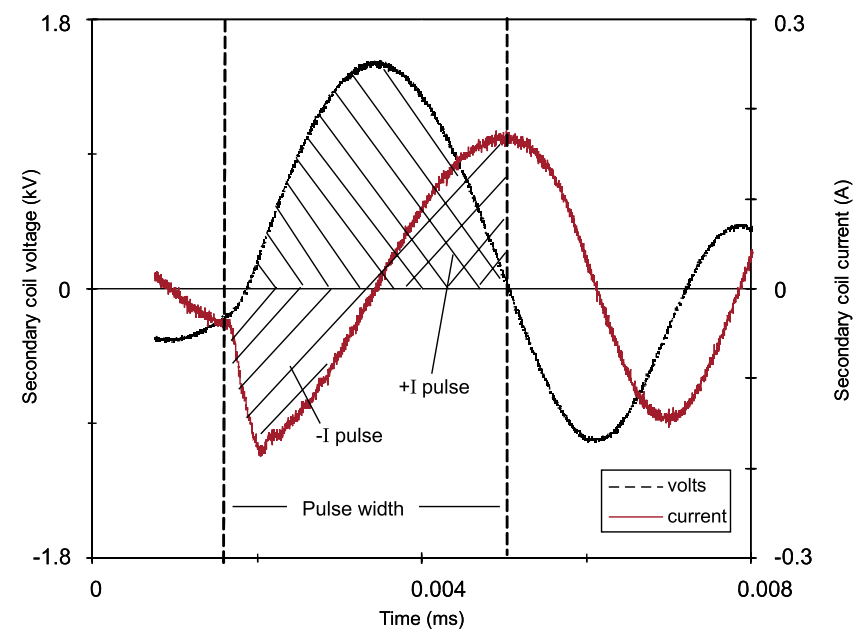

FIG. 5. (Color online) Schematic representation of estimation $V_{\mathrm{rms}}$ and $I_{\mathrm{rms}}$ values. 


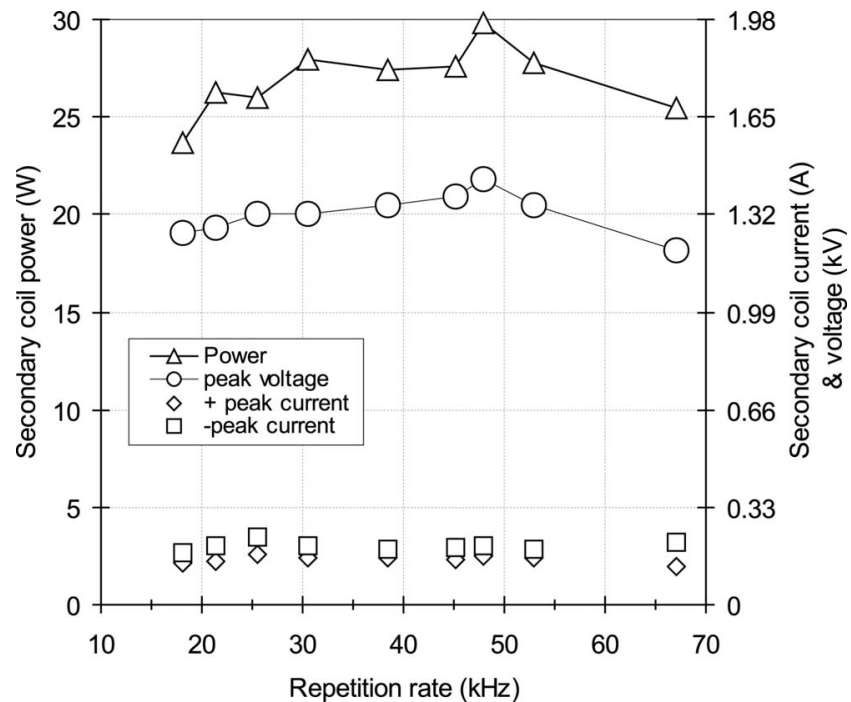

FIG. 6. Secondary coil peak voltage and peak current measurements as a function of pulse repetition rate at an argon gas flow rate of $5 \mathrm{slm}$. Calculated secondary power is shown for comparison.

good estimation of the average power delivered to the Flyback secondary coil. The values of $T$ and $B$ used are as follows: $T=1 \mu$ s and $B=3.5 \mu$ s for the voltage pulse, $T$ $=0.1 \mu \mathrm{s}$ and $B=1.75 \mu \mathrm{s}$ for the negative current pulse and $T=0.1 \mu \mathrm{s}$ and $B=3.5 \mu \mathrm{s}$ for the positive current pulse. As the primary current lags the primary voltage pulse by $90^{\circ}$, the positive current rms value is divided by half before summing the two $I_{\mathrm{rms}}$ values,

$$
X_{\mathrm{rms}}=X_{p} \times \sqrt{f \frac{[(B-T)+3 T]}{3}} .
$$

The voltage and current measurements plus the estimated power are shown in Fig. 6 for a given primary $3.5 \mu \mathrm{s}$ pulse the average power developed in the secondary coil increases from $23.6 \mathrm{~W}$ at $18 \mathrm{kHz}$ to $25.5 \mathrm{~W}$ at $70 \mathrm{kHz}$ with a maximum average power of $29.8 \mathrm{~W}$ at $48 \mathrm{kHz}$. Below and above this level the plasma cannot be maintained. The low PRR is consistent with other reported work where in the $1-20 \mathrm{kHz}$ range it has a weak influence on the breakdown voltage. ${ }^{15}$ The reason for the high pulse repetition rate limit is unclear from this graph. Clarification of this point is discussed in Sec. III D. A measurement of the second and third and fourth pulses indicates that they contribute approximately $3 \mathrm{~W}$ : giving a total of 33-37 W. Based on these measurements, the dc-to-pulse electrical power efficiency is $80 \%-99 \%$ in the $25-50 \mathrm{kHz}$ PRR.

Given that the argon gas flow is laminar within the dielectric barrier chamber, Netwon's law of heat transfer can provide an estimation of the forced convection heat lost via argon flow [see Eq. (6)].

$$
Q=\frac{\sigma_{a}}{L} \mathrm{Nu} A(\Delta T) \text {. }
$$

In Eq. (6), $Q$ is the rate of heat transfer, $\sigma_{a}$ is the thermal conductivity of the argon gas, $L$ is the length of the discharge volume, $A$ is the area of the discharge volume, $\mathrm{Nu}$ is the Nusselt number, and $\Delta T$ is the temperature difference between the dielectric barrier wall and the outside ambient temperature. Here the Nusselt number is defined as $0.664(\operatorname{Pr})^{1 / 3} \sqrt{\mathrm{Re}}$; where $\mathrm{Pr}$ is the Prandt number and is defined by the ratio of momentum diffusion rate to thermal diffusion rate (0.66 for argon), Re is the Reynolds number as defined in Eq. (4), and $\Delta T$ has a value of $50{ }^{\circ} \mathrm{C}$. Using this information the heat transfer rate by the argon gas approximates to 0.1 and $0.5 \mathrm{~W}$ for the flow rates used here.

\section{Discharge voltage and current performance}

This section examines the voltage and current waveform characteristics of the DBD as a function of six argon flow rates $(0,0.5,2,4,6$, and $8 \mathrm{slm})$ at a $50 \mathrm{kHz}$ PRR at a continuous applied dc power of $28.5 \mathrm{~W}(19 \mathrm{~V} \times 1.5 \mathrm{~A})$. These six data sets are displayed in Figs. 7(a)-7(c). The 0 slm data represent the same power condition but with no plasma present, the other five flow rate data set match different flow regions. Given $90 \%$ power transfer efficiency at 50 $\mathrm{kHz}$ this equates to $25.65 \mathrm{~W}$ at the secondary coil.

The first figure in this sequence [Fig. 7(a)] depicts the voltage and current waveforms for zero argon gas flow and no discharge present. The data also show that there is no distortion in the voltage and current waveforms apart from the damped oscillations. Here the primary voltage and current pulses reaching a maximum value of $1.5 \mathrm{kV}$ and $0.17 \mathrm{~A}$, and a comparison with the other five figures reveals that the voltage is at maximum and the current is at a minimum. From this it is concluded that the dielectric barrier is at an open circuit, with no discharge present.

Figure 7(b) represents a 2 slm argon flow rate where a single streamer projects from the dielectric barrier to the ground surface (see Fig. 4). The waveforms show that the primary pulse voltage has decreased to $1200 \mathrm{~V}$ and the distortion in both the voltage and current has increased. These distortions have the same zero-crossing-point time delay of $\sim 1 \mu \mathrm{s}$ as in Fig. 7(b), but the distortions are enlarged. The breakdown voltage is again $\sim 768 \mathrm{~V}$, but now it forms a separate peak from the main waveform with a magnitude of $30 \mathrm{~V}$. At this point in time, the current peak has a magnitude of $0.2 \mathrm{~A}$ with time duration of $0.04 \mu \mathrm{s}$. A comparison with the voltage and current data in Fig. 7(a) indicates that the resistance has fallen. After this pulse the current rises to 0.15 A where the second distortion has broadened to deform the waveform before reaching a maximum of $0.2 \mathrm{~A}$. The remaining current and voltage waveforms dissipate as a damped oscillation in a similar fashion to that in Figs. 7(a) and 7(b).

Figure 7(c) has argon gas flow rate of $6 \mathrm{slm}$ and is representative of the multifilament discharges as depicted in Fig. 4. The first feature of note in this triplet is that they all depict two voltage and current distortions in their respective rising voltage and current curves. These distortions are larger than observed in Figs. 7(b) and 7(c). The initial voltage distortion occurs at a breakdown voltage of approximately $768 \mathrm{~V}$ with a magnitude of $30 \mathrm{~V}$. This is followed by a second distortion at $1 \mathrm{kV}$ and $\sim 0.05 \mu$ s time delay. This second distortion has a magnitude of 60-90 V. Synchronized to these voltage distortions are two current peaks. The first peak has magnitudes of $0.14-0.1 \mathrm{~A}$ and the second peak having a greater magnitude of $0.2-0.25$ A. The time durations of these spikes are of the order of $\sim 0.03 \mu \mathrm{s}$. Note also, the deformations in the 

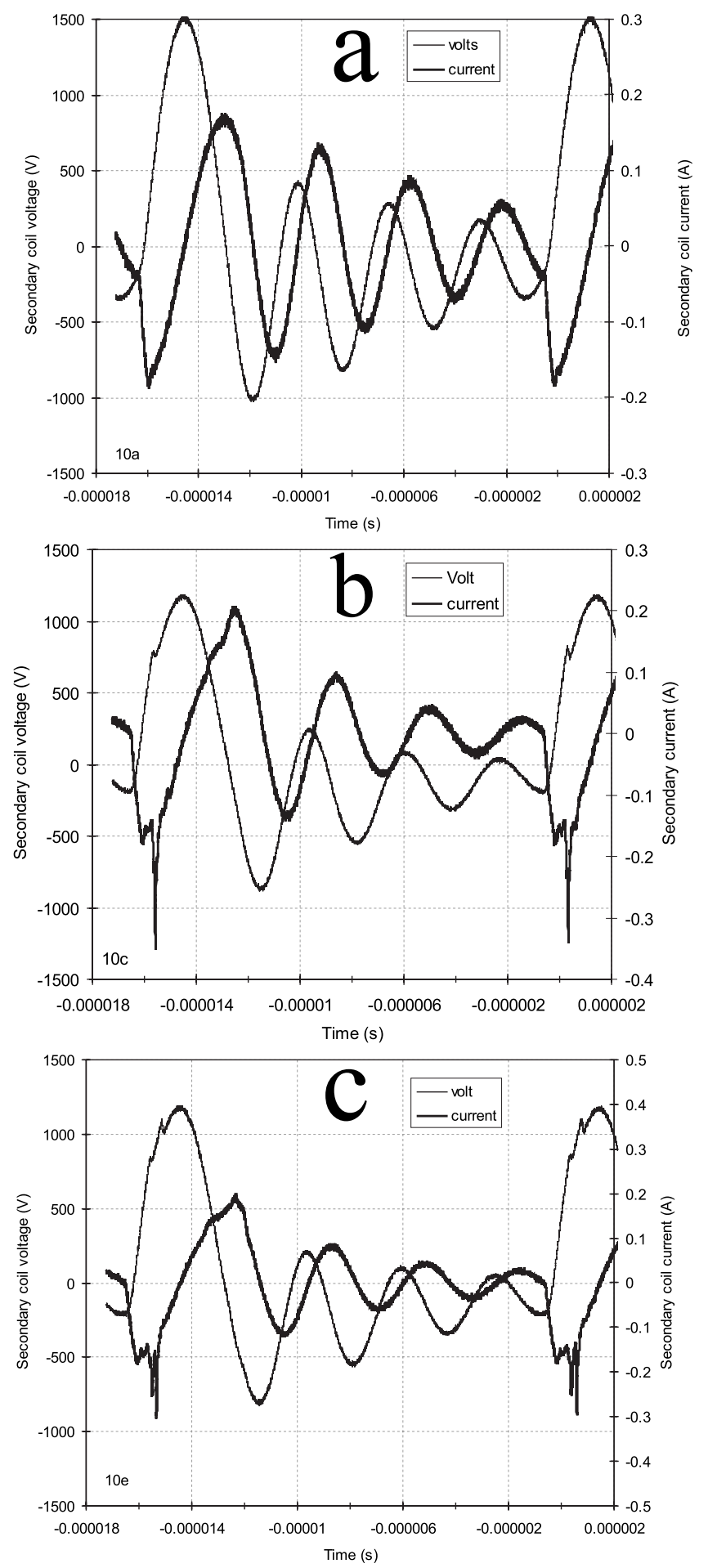

FIG. 7. Voltage and current waveforms at the secondary coil as a function of argon gas flow rate: $a=0 \mathrm{slm} ; b=2 \mathrm{slm} ; c=6 \mathrm{slm}$.

current waveform are more pronounced and with a corresponding deformation appearing in the voltage waveform.

Figures 7(b) and 7(c) show that the voltage and current waveforms are only distorted under discharge load conditions. From an electromagnetic viewpoint, the primary current spikes arise from a disproportional level of magnetomotive force $(\mathrm{mmf}=\mathrm{NI}$, where " $N$ " is the number of turns of wire in the coil and " $P$ " is the current through it) required to drive the discharge. Because $\mathrm{mmf}$ is proportional to current through the secondary coil, coil current increases dramati- cally at the breakdown voltage region. The second current distortion in the latter part of the current waveform needs to be analyzed with regard to the Flyback magnetic current mode of operation: continuous mode, or discontinuous mode. For this, a comparison of the primary coil current and the secondary coil current needs to be made. This comparison is made in Sec. III D.

The average electrical power within the discharge pulse(s) over a time period of $1 \mathrm{~s}$ is estimated from the product of the current and voltage rms values as described in Eq. (4), where the pulse shape values used are $B=0.3 \mu$ s and $T=0.1 \mu \mathrm{s}$, for a $50 \mathrm{kHz}$ PRR. Table II tabulates the measured peak values, the calculated rms, and the average power values for Figs. 7(b) and 7(c). With argon flow rates of 0.5, 2, 4, 6, and $8 \mathrm{slm}$ the combined current and voltage spike power is calculated. In the last row of Table II the $10 \mathrm{slm}$ power data are included. The average power density $\left(\mathrm{mW} / \mathrm{cm}^{3}\right)$ for each row is given in column 4 . These tabulated results show the power in the discharge power increases with gas flow from $3 \mathrm{~mW}$ at $0.5 \mathrm{slm}$ to $140 \mathrm{~mW}$ at 8 slm.

A graphical representation of the calculated average power is shown in Fig. 8; the data for no argon gas flow is also shown for completeness. A linear trend line has been applied to observe the data spread and $y$ error bars applied until all data points cross the trend line. A $\pm 20 \%$ error achieves this goal. From this process it can be shown that the discharge power density increases linearly with argon gas flow at a rate of $75 \pm 20 \% \mathrm{~mW} / \mathrm{cm}^{3}$ per $1 \mathrm{slm}$. In the $4-6$ slm range the average power is $107 \mathrm{~mW}[(94+120) / 2]$ and increases to $755 \mathrm{~mW}$ at $10 \mathrm{slm}$. These values show that the power transfer efficiency from the secondary coil to the discharge increases with gas flow, and has increased from $0.01 \%$ to $0.65 \%$. However, the power achieved using the Flyback circuit is lower than reported for similar helium flow rates in Ref. 10 when a $13.56 \mathrm{MHz}$ drive frequency is applied. This is to be expected considering that the drive is 271 times lower in drive frequency and the argon process requires a stronger electric field compared to the helium ionization process. For comparison, Fig. 8 plots both the neutral gas residence time (Fig. 4) and power density as a function of argon flow rate. The comparative data reveal that the power density behavior is inversely proportional to the neutral gas residence. In effect the ionized argon gas is acting as a dissipative (energy consuming) load, where the load increases with the amount of gas passing through the discharge volume.

\section{Discontinuous and continuous modes of operation}

In Sec. II A (Fig. 2) and in Sec. III C the concept of continuous and discontinuous current mode operations was introduced. In the continuous current mode operation, ripple current, and ac winding loss are mainly dominant, whereas in the discontinuous mode, ac winding loss is the main source of energy loss. Therefore the impact of these two modes upon power transfer efficiency from the secondary coil to the discharge need to be understood in this context. Figure 9 shows typical primary and secondary magnetic current waveforms for argon discharge production. Here it can be seen 
TABLE II. Discharge electrical performance as a function of argon flow rate at a secondary coil power of $28.2 \mathrm{~W}$.

\begin{tabular}{lccccc}
\hline \hline $\begin{array}{l}\text { Flow rate } \\
(\mathrm{slm})\end{array}$ & $\begin{array}{c}\text { Peak voltage } \\
\left(V_{\text {rms }}\right)\end{array}$ & $\begin{array}{c}\text { Peak current } \\
\left(I_{\text {rms }}\right)\end{array}$ & $\begin{array}{c}\text { Power } \\
(\mathrm{mW})\end{array}$ & $\begin{array}{c}\text { Power efficiency } \\
(\%)\end{array}$ & $\begin{array}{r}\text { Power density } \\
\left(\mathrm{mW} / \mathrm{cm}^{3}\right)\end{array}$ \\
\hline 0.5 & $\sim 10(\sim 0.77)$ & $0.05(0.004)$ & 3 & 0.01 & 12 \\
2 & $30(2.324)$ & $0.2(0.015)$ & 35 & 0.12 & 140 \\
4 (first spike) & $30(2.324)$ & $0.14(0.011)$ & 24 & & 96 \\
4 (second spike) & $60(4.648)$ & $0.2(0.015)$ & 70 & & 280 \\
4 (combined power) & & & 94 & 0.33 & 376 \\
6 (first spike) & $60(4.648)$ & $0.1(0.008)$ & 37 & & 332 \\
6 (second spike) & $90(6.91)$ & $0.15(0.012)$ & 83 & & 480 \\
6 (combined power) & & & 120 & 0.43 & 148 \\
8 (first spike) & $60(4.648)$ & $0.1(0.008)$ & 37 & & 412 \\
8 (second spike) & $90(6.51)$ & $0.2(0.015)$ & 103 & & 560 \\
8 (combined power) & & & 140 & 0.5 & 755 \\
10 (first spike) & $90(6.51)$ & $0.4(0.029)$ & 188 & 0.65 & \\
\hline \hline
\end{tabular}

that at the timer turn-off trigger point the two magnetic currents are out of phase, however, the instantaneous primary coil current does not return to zero until the end of second current pulse. This equates to a time period $\sim 6 \mu$ s. Note, the time period where the primary magnetic current is stable at $\sim 1 A$ is aligned with the deformation of the secondary coil current waveform [see Figs. 7(b) and 7(c)]. In this time region not all the energy is being transferred between the primary and secondary. Under these conditions the mmf cannot be sustained; hence the secondary magnetic waveform in this region is deformed. By definition the Flyback transformer is operating in the continuous magnetic current mode. It is only after $\sim 20 \mu$ s that both currents truly cross into the discontinuous mode of operation. This boundary crossover equates to a $50 \mathrm{kHz}$ PRR. Operating with a timer off period shorter than this, or faster repetition rates, it is observed that the power deposited within the discharge begins to fall (see Fig. 6). From this observation it may be empirically concluded that the mode of operation and the timer off period, rather than power supply in this case, provides the limit to the

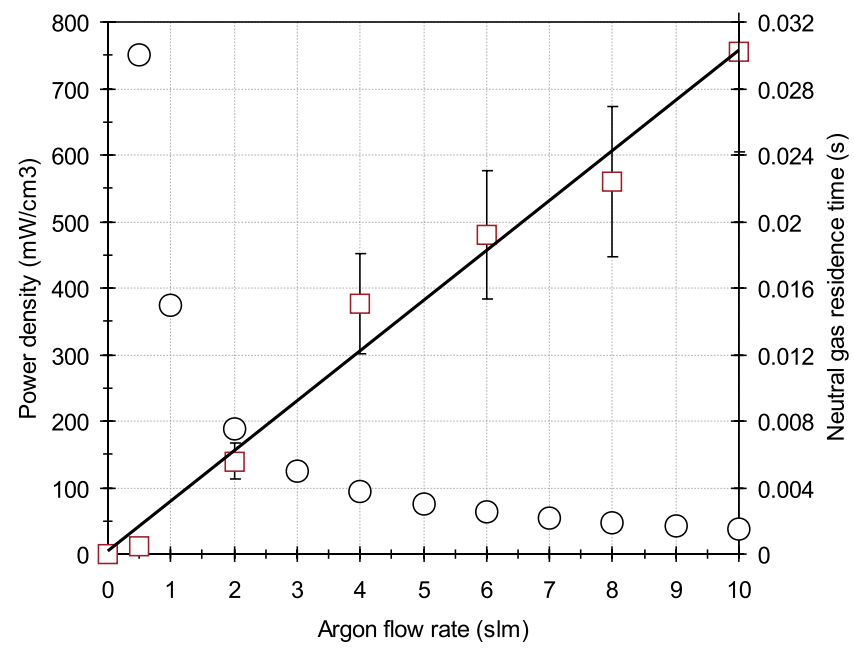

FIG. 8. (Color online) Discharge power density and neutral gas residence time as a function of argon flow rate. Primary pulse $=21 \mathrm{~W}$. Trend line with $\pm 20 \%$ error bars added to aid the eye. power transfer efficiency of the Flyback circuit, i.e., $50 \mathrm{kHz}$ PRR at a $50 \%$ duty cycle.

\section{E. Optical emission spectroscopy}

The optical emission spectroscopy (OES) studies of the argon expanding plume into ambient air have been performed. In this study an Ocean optics spectrometer ${ }^{\circledR}$ was used. The instrument was set to an integration time of $0.3 \mathrm{~s}$ and wavelength span between 250 and $850 \mathrm{~nm}$ with an effective resolution of $1 \mathrm{~nm}$. A Quartz optical fiber and collimating lens was used to image the plume at a distance of 5 $\mathrm{cm}$. To remove the effect of local regions of streamers and filaments within the expanding plume, the plume was axially imaged through a microscope slide which also acts as a dielectric and could, for example, mimic a microbial biofilm.

Figure 10 shows OES spectra of the argon expending plume at a flow rate of $2.5 \mathrm{slm}$. The power parameters are $200 \mathrm{~mW} / \mathrm{cm}^{3}$ and $\sim 50 \mathrm{kHz}$ PRR. The spectrum shows that no positive argon ions are detected; however, there is an abundance of neutral argon atoms with emission wavelengths between 415.859 and $852.144 \mathrm{~nm}$. The next abun-

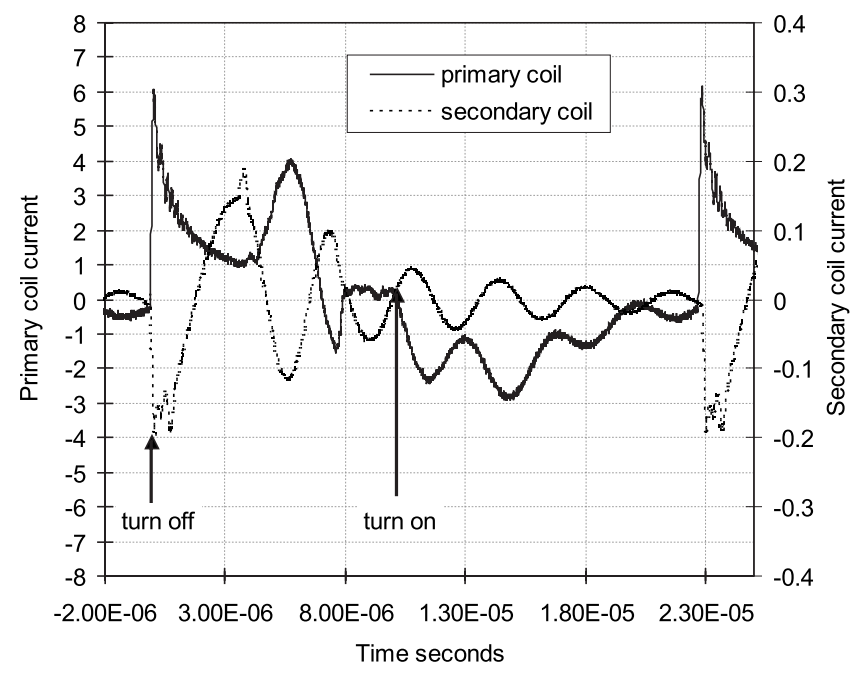

FIG. 9. Comparison between primary and secondary coil currents. 


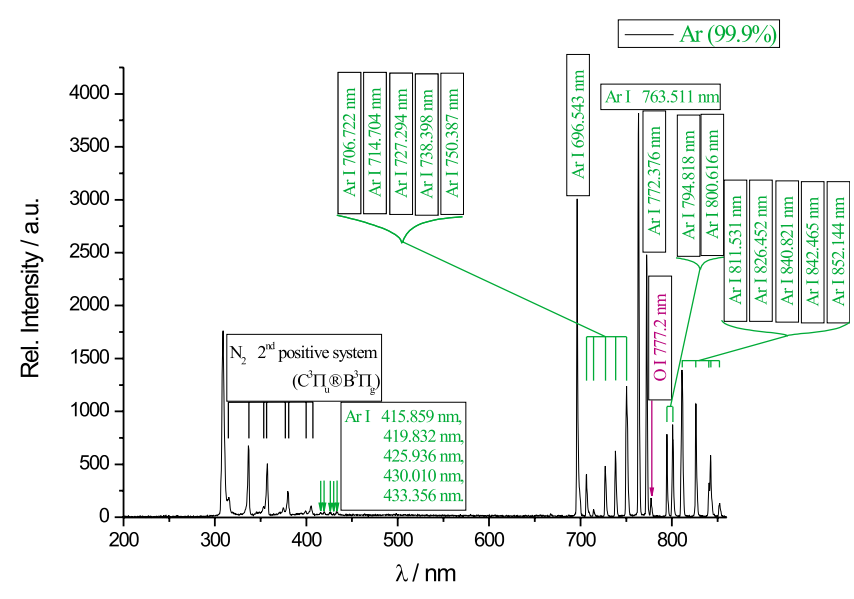

FIG. 10. (Color online) OES measurement of atmospheric argon discharge.

dant molecule is diatomic nitrogen with an energy level assigned to the second positive system $\left(C^{3} \Pi_{u}\right.$ $\left.\rightarrow B^{3} \Pi_{g}: 11.1 \mathrm{eV}\right) .^{20}$ This emission system is similar to those measured in atmospheric pressure nitrogen discharges. ${ }^{9}$ Two other emission lines are observed, one at 308 and 777 $\mathrm{nm}$. The 308 emission may be attributed to either the hydroxyl $(\mathrm{OH})$ molecular band $\left(\mathrm{A}^{2} \Sigma^{+}, v=0 \rightarrow X^{2} \Pi, v^{\prime}\right.$ $=0,306-310 \mathrm{~nm}),{ }^{21}$ or atomic aluminum $(309 \mathrm{~nm})$. In the latter case, the aluminum originates from low energy argon or nitrogen ions ${ }^{22}$ sputtering off the inner surface of the chamber $\mathrm{Al}_{2} \mathrm{O}_{3}$ wall. The OES equipment used here has insufficient wavelength resolution to resolve this issue; however, as the 309 emission is approximately ten times greater than the atomic oxygen emission line at $777 \mathrm{~nm}$, aluminum is thought to be the most likely candidate.

A comparison with a helium discharge within the single chamber DBD at similar gas and power conditions reveals that the expanding plume chemistry is very different. This is shown in Fig. 11. In general, the diatomic nitrogen second positive system and the diatomic nitrogen positive ions $\left(B^{2} \Sigma_{u}^{+} \rightarrow X^{2} \Sigma_{g}^{+}: 18.7 \mathrm{eV}\right)$ are observed. These systems are similar in appearance to helium plumes studied in Ref. 7 and 15. In Ref. 15 the appearance of the diatomic nitrogen positive system has been attributed to the high pressure Penning

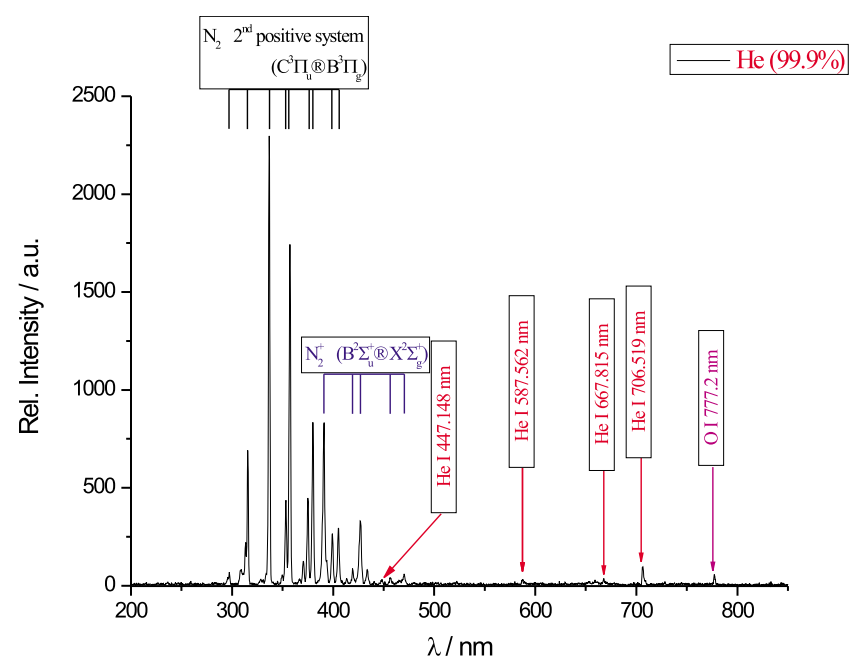

FIG. 11. (Color online) OES measurement of atmospheric helium discharge. ionization process upon energetic helium atoms as represented in

$$
\mathrm{He}\left(2{ }^{3} S\right)+\mathrm{N}_{2} \rightarrow \mathrm{He}+\mathrm{N}_{2}^{+}+e .
$$

OES measurements show that the $\mathrm{N}_{2}: 336.7$ and the $\mathrm{N}_{2}^{+}: 391.1 \mathrm{~nm}$ emission lines increases with flow rate, PRR, and power density and correlate with the low transitional energy helium 706.3 emission lines. This observation is a good indicator of the high pressure Penning ionization process that operates in the helium expanding plume.

The atomic helium most insensitive spectral line in our spectrum is at $706.519 \mathrm{~nm}$. This emission is observed, when electron from excited state $3 s\left({ }^{3} P_{1}\right)$ finished at one of metastable helium level i.e., at $2 p\left({ }^{3} P_{0,1,2}\right)$ with energy of 20.96 $\mathrm{eV}$. The upper energy level of this line is at $22.72 \mathrm{eV}$, i.e., $3 s\left({ }^{3} S_{1}\right)$. In comparison with other atomic helium spectral lines recorded with the experiment setup, the $706.519 \mathrm{~nm}$ has the lower upper energy state, therefore it is observed much easier then the other helium spectral lines. The intensity of He I 706 spectral line show very interested behavior. Namely, plotting spectral intensity of He I 706 versus PRR frequencies, we have observed a local minimums for each gas flow at a single frequency. The observed frequency is moved to a shorter frequency with increasing helium flow, see Fig. 12. Moreover at the same moment we record the boost in intensity of $\mathrm{N}_{2} 336.7$ molecular band. It is well know that the helium metastable plays an important role in plasma kinetics of gas discharges like ours. ${ }^{7}$ Decreasing He I 706 spectral line intensity means a decreasing in the number of helium atoms in the metastable state. This leads to a decrease in dissociation of $\mathrm{N}_{2}$ molecule by collision with the helium metastable atoms (which is one of main mechanism for dissociation), which turned out at the end into the boost of $\mathrm{N}_{2}$ signal recorded at $336.7 \mathrm{~nm}$. In the case of the argon discharge, metastable argon atoms do not have the same role as helium metastable atoms, mainly because they have almost two times less energy than helium metastable atoms and the fact that the mass of an argon atom is ten times bigger than helium. This is also related to velocity of a metastable atom. Nevertheless we have recorded the intensity of several argon spectral lines that have lower energy level at the metastable state.

\section{CONCLUSION}

The development of an atmospheric pressure Flyback DBD has been performed. The issues addressed include external electrical drive circuitry, Flyback transformer design, mechanical construction of chamber, fluid mechanics of the working gas, electrical power consumption, and OES of the expanding plume.

It has been shown that self-resonant and external drive circuitry can drive a Flyback transformer to sustain both helium and argon DBDs. The power switching mechanism for both these circuits utilizes a MOSFET.

Voltage and current monitoring on the Flyback primary and secondary coils characterizes the DBD in terms of continuous de supply to pulse power transfer efficiency, discharge power, discharge power density, and current mode of operation. For a continuous dc power of $30 \mathrm{~W}$ and a selected 

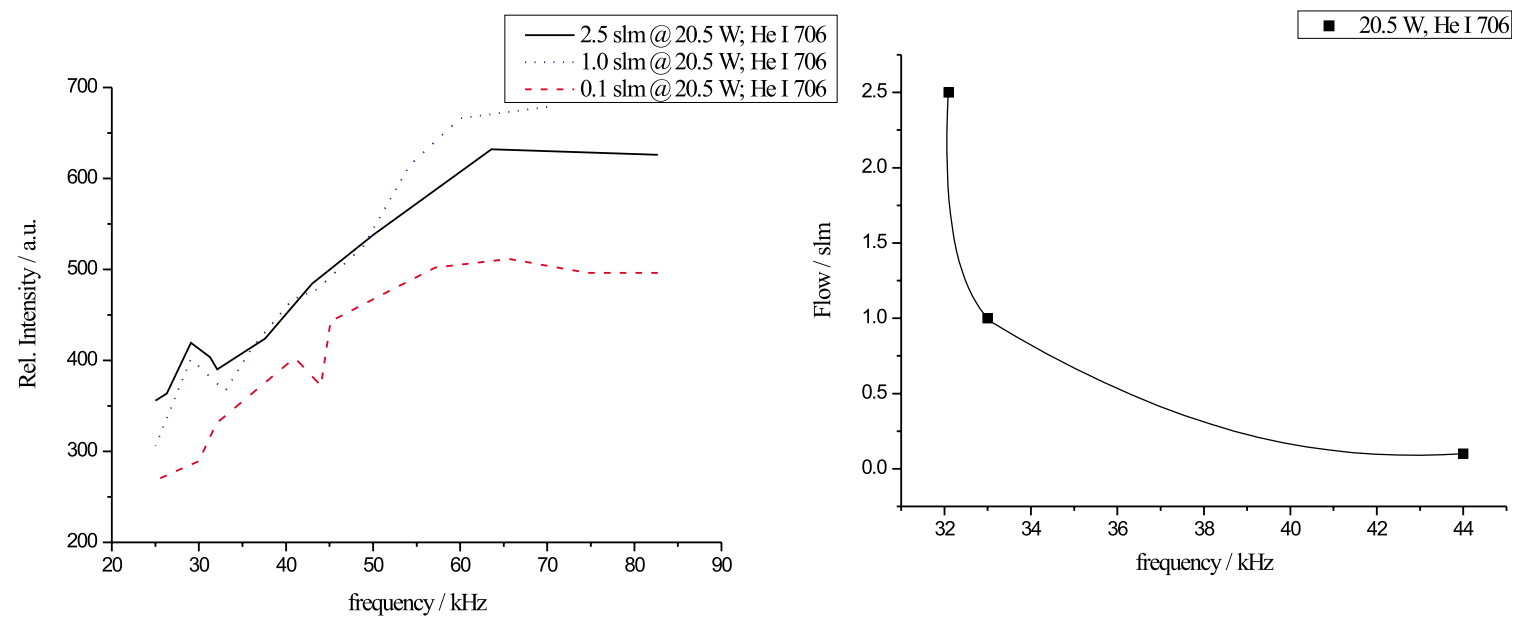

FIG. 12. (Color online) Intensity of He I $706.52 \mathrm{~nm}$ spectral line vs frequency (left figure). The behavior of local minimum (in emission of He I 706 spectral line) with respect of gas flow and frequency (right figure).

$50 \mathrm{kHz}$ PRR, the argon discharge power, and power density, increases linearly at a rate of $75 \pm 10 \% \mathrm{~mW} / \mathrm{cm}^{3}$ per $1 \mathrm{slm}$, between 0.5 and $10 \mathrm{slm}$. This rate dependency is shown to be inversely proportional to the neutral gas residence time. These measurements reveal that an argon discharge is a dissipative (energy consuming) load.

Electrical energy transfer efficiency between the dc power supply and the secondary coil is in the range of $90 \%$ and the secondary coil to discharge efficiency is in the range of $0.1 \%-0.65 \%$ within the primary pulse. A fluid mechanics analysis of the energy transfer by the neutral argon gas flow suggests a similar energy loss rate to that of the discharge process. The remaining energy loss $(\sim 89 \%)$ is dissipated in the dielectric barrier chamber as heat.

Scaling up from a single to triple DBD chamber indicates that equal gas flow supply to the chambers, rather than dc power supply, needs to be considered. The scaling up also points to the possibility that flat areas of $\sim 50 \mathrm{~mm}$ in diameter can be illuminated from a plume that originates from a multiple chamber $(\sim 20)$ DBD configuration.

Optical emission studies reveal that argon and helium discharges are highly quenched in the expanding plasma plume where ambient air climatically controls the quenching process. In the case of helium discharge, water clusters, diatomic nitrogen second positive system, and diatomic nitrogen ions are produced; for an argon discharge, the diatomic nitrogen second positive system and a range of argon neutrals are produced. In general, for the helium discharge, the plume chemistry may be attributed to a high pressure Penning ionization process. This Penning process is absent in the argon plume discharge, presumably due to argon having insufficient energy to populate the $\mathrm{N}_{2}^{+}$state. The ability to climatically control plume chemistry allows a limited control of the reaction mechanism at a target surface. This controlling mechanism is a desirable attribute in the destruction of microbial pathogens on contaminated surfaces.

\section{ACKNOWLEDGMENTS}

This work was supported by the Enterprise Ireland under Grant No. CFTD/7/IT/304. V. Milosavljević was grateful to project "The use of non invasive OES as method for the determination of gas species concentration and the main plasma parameters in industrial plasma processing equipment" funded by the Enterprise Ireland and to the Ministry of Science and Environment Protection of the Republic of Serbia for Grant No. OI141031 "Nonlinear dynamical phenomena in photorefractive media, liquid crystals, plasmas and left-handed materials."

${ }^{1}$ B. Eliasson, M. Hirth, and U. Kogelschatz, J. Phys. D 20, 1421 (1987).

${ }^{2}$ B. Pashaie, S. K. Dhali, and F. Honea, J. Phys. D 27, 2107 (1994).

${ }^{3}$ Q. Chen, Y. Zhang, E. Han, and Y. Ge, Plasma Sources Sci. Technol. 14, 670 (2005).

${ }^{4}$ M. Laroussi, IEEE Trans. Plasma Sci. 30, 1409 (2002).

${ }^{5}$ I. E. Kieft, E. P. van den Laan, and E. Stoffels, New J. Phys. 6, 149 (2004).

${ }^{6}$ E. Stoffels, I. E. Kieft, R. E. J. Sladek, L. J. M van den Bedem, E. P. van den Laan, and M. Steinbuch, Plasma Sources Sci. Technol. 15, S169 (2006).

${ }^{7}$ J. L. Walsh, J. J. Shi, and M. G. Kong, Appl. Phys. Lett. 88, 17150 (2006).

${ }^{8}$ S. D. Anghel, and A. Simon, Meas. Sci. Technol. 18, 2642 (2007).

${ }^{9}$ E. Panousis, F. Clments, J.-F. Loiseau, N. Spyrou, B. Held, M. Thomachot, and L. Marlin, Plasma Sources Sci. Technol. 15, 828 (2006).

${ }^{10}$ M. Kong and X. T. Dang, IEEE Trans. Plasma Sci. 31, 7 (2003).

${ }^{11}$ N. J. Rowan, S. J. MacGregor, J. G. Anderson, D. Cameron, and O. Farish, Appl. Environ. Microbiol. 67, 2833 (2001).

${ }^{12}$ A. M. Aniplov, E. M. Barkhudarov, N. Christofi, V. A. Kop'ev, I. A. Kossyi, M. I. Taktakishvili, and Y. V. Zadiraka, J. Water Health 4, 267 (2004).

${ }^{13}$ V. J. Law, J. Lawler, and S. Daniels, Vacuum 82, 514 (2008).

${ }^{14}$ V. J. Law, Vacuum 82, 630 (2008).

${ }^{15}$ A. Sublet, C. Ding, J.-L. Dorier, Ch. Hollenstein, P. Fayet, and F. Coursimault, Plasma Sources Sci. Technol. 15, 627 (2006).

${ }^{16}$ T. Nozaki, Y. Unno, and K. Okazaki, Plasma Sources Sci. Technol. 11, 431 (2002).

${ }^{17}$ Y. Aranda-Gonzalvo, D. L. Seymour, C. L. Greenwood, J. A. Rees, T. D. Whitmore, S. Daniels, and V. J. Law, Proceedings of the 18th ISPC (poster 30P-59, abstract No. 137), Kyoto, Japan, 2007 (unpublished).

${ }^{18} \mathrm{~T}$. Whitmore, private communication (2007).

${ }^{19}$ B. Chapman, Glow Discharge Process (Wiley, New York, 1980), Chap. 1, pp. 14-19.

${ }^{20}$ R. C. Pankhurst, Proc. Phys. Soc. 52, 388 (1940).

${ }^{21}$ S. Y. Moon and W. Choe, Spectrochim. Acta, Part B 58, 249 (2003).

${ }^{22}$ J. P. Holgado, F. Yubero, A. Cordn, F. Gracia, A. R. Gonzlez-Elipe, and J. Avila, Solid State Commun. 128, 235 (2003). 
Review of Scientific Instruments is copyrighted by the American Institute of Physics (AIP). Redistribution of journal material is subject to the AIP online journal license and/or AIP copyright. For more information, see http://ojps.aip.org/rsio/rsicr.jsp 